\title{
Research on Equipment in Orbit Early Warning Subsystem
}

\author{
Yuan Sun ${ }^{1,}$, , Haifeng Yang 2 , b \\ ${ }^{1}$ Naval Aeronautical and Astronutical University, Shandong 264001, China \\ ${ }^{2} S h a n d o n g$ Institute of Aerospace Electric Technology, Shandong 264003, China \\ asunyuan1208@126.com, bxdyhf@126.com
}

Keywords: spacecraft, early warning, on-board faults, satellite, forecast.

\begin{abstract}
When spacecraft is working in orbit, massive data that reflects status of spacecraft is produced. This information truly reflects the changing process of observe and control events and faults, which can be used on the early warning of the status development trend of spacecraft. Therefore, analyzing the feature of in orbit data and on which basis predicting its development trend, and making early warning of spacecraft's working status according to the prediction information make it possible to discover abnormal changes of spacecraft's features in early phase and solve problems in time so that to avoid major failure and reduce the risk of satellite's in orbit working. Using the next-generation communication satellite as an example, the early warning subsystem for communication satellite is designed. According to engineering application, using typical voltage and temperature data as an example, the early warning result is given, which shows the early warning effect is good.
\end{abstract}

\section{Introduction}

When Spacecraft is working in orbit, after long-term working in the space environment, its performance and function will change with the factors of time, space environment, control events and faults. These changes are reflected by the telemetry data, so that we can analyze the law of the data changes of the spacecraft in orbit. On the basis of analyzing the telemetry data, we can make early warning according to the spacecraft performance and we can detect the abnormal changes of the spacecraft for timely and effective treatment to avoid major failure that may occur, and reduce the risk of spacecraft in orbit.

In this thesis, we design early warning system of telemetry data that can be used on satellite platform, by which, we can detect the abnormal changes of satellite performance, so that the satellite will make early warnings. In addition, it is of important significance to discover and conclude the law of the telemetry data changes according to the long-term forecast satellite historical telemetry data to make sure that the satellite will perform safe and stable in orbit and we can make health assessment and performance research of the satellite and improve the satellite designs.

\section{Research on Satellite System}

\subsection{Uniqueness of the satellite system}

Satellite system has its particularity, which is mainly reflected in the following four aspects:

The system cannot be maintained. The system is exposed to complex electromagnetic environment, and it is easy to be disturbed; System capacity is low, usually there is only one system; Even if the fault occurred, the system will keep on working as far as possible.

These special causes lead fault to satellite system and make great risk to safe operation. The traditional satellite needs manual management on the ground station, because of the limited communication, it cannot make real-time control. With the demand of the real time and reliability of satellite management, it is necessary to reduce the ground manual management and improve satellite autonomous control and autonomous fault management. The FDIR system designed to have the ability of fault detection, fault isolation and fault isolation, can make real time monitor and fault detection of 
the whole state of the satellite, locate the fault and take the measure of reconstructing to ensure the safe and reliable operation of the satellite.

\subsection{Research on early warning system}

The FDIR is used to monitor and deal with the satellite when the fault had happened, but some fault may have caused irreparable losses, so, before the fault has occurred, it is an effective method to take some measures to avoid the fault occurrence to manage a satellite in orbit.

It is an important direction for the satellite in orbit management to find the law that when satellite in orbit, its performance and functionality will change with the time and space environment, control events and failure factors according to the analysis of the in orbit satellite telemetry data. According to the analysis telemetry data in orbit and forecast the future development laws of the telemetry data, and applied to the forecasting of the data, it can make warning of the abnormal state the satellite may exist. It is a new direction of data analysis. Satellite telemetry data early warning system has achieved the function of telemetry data trend forecasting and early warning in ground support systems, and accumulates some experience and methods for satellite telemetry data processing, forecasting and early warning. Trend forecasting system run on the satellite avoids a lot of disturbance, it can get more real-time, accurate and extensive telemetry data to predict the trend of telemetry data, also it can early detect the abnormal changes of satellite performance, so that satellite early warning will work.

Through the analysis of satellite system, we put up the spacecraft in orbit early warning system, the system completes the forecasting and early warning tasks in all parameters of the satellite which has significant variation of telemetry parameter, mainly for the analog quantity, temperature and some other types.

1) Real time forecasting function: using of the past satellite telemetry data, we take accordance methods to make real time forecast for telemetry parameters.

2) Early warning function: Comparing the telemetry parameters predictive value, confidence degree and the theory threshold the user set, when the forecasting value deviates from the confidence level or beyond the theoretical threshold, the warning information is given in time.

Early warning system uses modular design, it consists by the parts as follows: the data receiving and distributing subsystem, telemetry parameter forecasting subsystem, early warning management subsystem, task allocation and the center control subsystem and in orbit telemetry database system. All the subsystems complete the on orbit telemetry data trend forecast and early warning function. The architecture is as Fig 1.

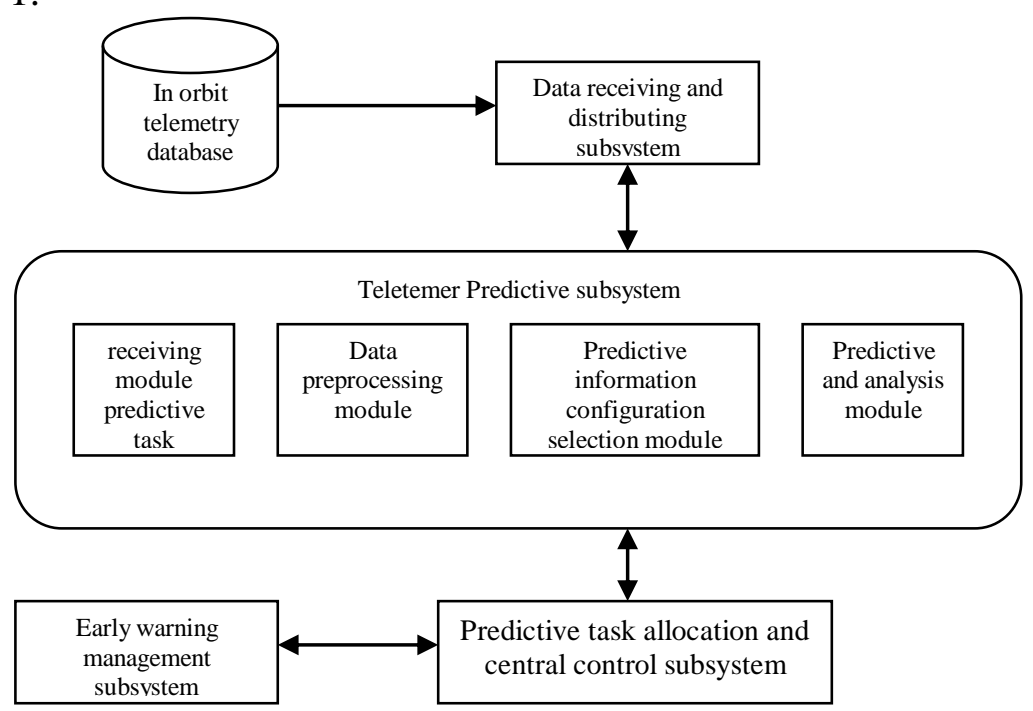

Fig 1 general planning for the system

3) Data receiving and distributing subsystem: early warning system and exchange the information with telemetry database in orbit.

4) Telemetry parameter forecast subsystem: predicting, getting historical telemetry data, preprocessing of historical telemetry data, forecasting the task allocation, forecasting algorithm model configuration, forecasting analysis and information output. 
5) Early warning management subsystem: completing the forecast data acquisition, comparison and analysis, when the forecasted values deviating from the confidence or beyond the theoretical threshold, the real-time warning information is given.

6) Task allocation and the center control subsystem: Responsible for the control and scheduling of the whole system, the forecast results are given to the control terminal, which is used in orbit management, fault diagnosis and so on.

7) In orbit telemetry database system: Storage and management of the satellite in orbit telemetry data.

\section{Research on implementation method of trend forecast}

\subsection{Data preprocessing method}

In the process of collecting and distributing data of the in orbit telemetry data, the data is affected by the acquisition system, encoding, communication and some other facts, and there will be some outliers. The existences of outliers will affluence the results of data forecast. Therefore, it is necessary to preprocess the telemetry parameters data and eliminate the outliers. Analyzing the telemetry data to be forecast, we can use the following several outlier elimination method on the different characteristics of data preprocess, to eliminate the outliers.

1) Tucci median outlier elimination method: Processing data information changes stable.

2) Four point smoothing method for outliers: Processing data information changes smoothly.

3) Standard variance ratio method: Processing data information has strong periodicity.

4) Standard variance ratio method of plot: Processing data information has many uncertain factors.

\subsection{Classification and comparison of forecasting methods}

Trend forecasting methods can be divided into the following three categories: trend forecasting technology based on telemetry data, trend forecasting technology based on physical mode and trend forecasting technology based on knowledge, among which, the trend forecasting technology based on telemetry data can be divided into the trend forecasting of telemetry data based on mathematical model and trend forecasting technology of telemetry data based on intelligent algorithm. Table 1 compares and analyses the applicable objects, features and applications of 3 kinds of trend forecasting methods.

3 kinds of trend forecasting methods have their advantages and disadvantages; the trend forecasting technology based on physical mode needs a precise mathematical model, trend forecasting technology based on physical mode and trend forecasting technology based on knowledge is limited by expert knowledge acquisition. These two methods are seldom used in engineering practice, therefore the satellite trend forecast system uses the method of trend forecasting technology based on telemetry data.

In order to meet the needs of the satellite in orbit data analysis and trend forecasting, the forecasting system of the trend forecast system provides a variety of typical methods based on the forecast of the telemetry data. In the practical application of in orbit management, the appropriate forecasting method is chosen according to the data in orbit and parameters characteristic. Taking temperature and voltage of two typical telemetry parameters for example, based on engineering practice, the trend forecast method such as the least square method, the method based on Fourier and neural network will lead to good forecast effect.

Table 1 Comparison of all kinds of trend forecasting methods 


\begin{tabular}{|c|c|c|c|c|}
\hline \multicolumn{2}{|c|}{ Prediction type } & $\begin{array}{l}\text { Typical } \\
\text { method }\end{array}$ & Applicable object & Characteristic \\
\hline \multirow{2}{*}{$\begin{array}{l}\text { Based on } \\
\text { telemetry } \\
\text { data }\end{array}$} & $\begin{array}{c}\text { Mathematical } \\
\text { model }\end{array}$ & $\begin{array}{l}\text { Least Square } \\
\text { Method, } \\
\text { ARMA } \\
\text { model, Based } \\
\text { on Fourier } \\
\text { method and } \\
\text { etc }\end{array}$ & \multirow{2}{*}{$\begin{array}{l}\text { The test data } \\
\text { sequence } \\
\text { changes with } \\
\text { time and it has } \\
\text { certain rules. }\end{array}$} & \multirow{2}{*}{$\begin{array}{l}\text { The method is based on the } \\
\text { acquisition of the time series of } \\
\text { teltmetry data, and it is easy to } \\
\text { implement, and it is not necessary } \\
\text { to describe the physical model of } \\
\text { the device, and it does not require a } \\
\text { priori knowledge of experts in } \\
\text { various fields. }\end{array}$} \\
\hline & $\begin{array}{l}\text { Intelligent } \\
\text { algorithm }\end{array}$ & $\begin{array}{c}\text { Neural } \\
\text { network, } \\
\text { Support vector } \\
\text { machine, } \\
\text { SARIMA and } \\
\text { etc }\end{array}$ & & \\
\hline \multicolumn{2}{|c|}{ Based on physical model } & $\begin{array}{l}\text { Kalman Wave } \\
\text { filtering }\end{array}$ & $\begin{array}{l}\text { System model is } \\
\text { known, accurate } \\
\text { system model } \\
\text { can be } \\
\text { established. }\end{array}$ & $\begin{array}{l}\text { Although the proposed method has } \\
\text { higher prediction accuracy, it is } \\
\text { difficult to establish a precise } \\
\text { mathematical model, so it has little } \\
\text { application. }\end{array}$ \\
\hline \multicolumn{2}{|c|}{ Based on knowledge } & $\begin{array}{c}\text { Expert } \\
\text { system, Fuzzy } \\
\text { logic }\end{array}$ & $\begin{array}{l}\text { Be rich in expert } \\
\text { knowledge, a } \\
\text { complete } \\
\text { knowledge base } \\
\text { can be } \\
\text { established. }\end{array}$ & $\begin{array}{l}\text { The method can utilize the expert } \\
\text { knowledge and experience in the } \\
\text { field of object system, but it is } \\
\text { limited to incomplete knowledge } \\
\text { acquisition of expert knowledge, it } \\
\text { is only suitable for qualitative } \\
\text { reasoning and not for quantitative } \\
\text { calculation, so its application in } \\
\text { practice is Limited. }\end{array}$ \\
\hline
\end{tabular}

\subsection{Forecasting model and algorithm}

In the forecasting process, the least square polynomial model, SumSin model, Fourier Series model, Exponent model, Gauss model and Neural network model are used.

1) Least squares polynomial model

On the principle, the polynomial model can well fit the data with periodic law, and the low order polynomial model, especially the slow variation to the exponential form. Take the 4 order model as an example, the model expression is as follows:

$$
y=\Phi(x, t)=a_{1} t+a_{2} t^{2}+a_{3} t^{3}+a_{4} t^{4}
$$

In the model expression, $x=\left(a_{1}, a_{2}, a_{3}, a_{4}\right)$ is the model parameters to be solved, the number of model parameters $n=4, y$ is telemetry sampling data, $t$ is corresponding sampling time sequence.

2) SumSin model

SumSin model is sin function and model, the model can well fit the data of various types of periodic law. Take the 6 order model as an example, the model expression is as follows:

$$
\begin{aligned}
y=\Phi(x, t)= & a_{1} \sin \left(b_{1} t+c_{1}\right)+a_{2} \sin \left(b_{2} t+c_{2}\right)+a_{3} \sin \left(b_{3} t+c_{3}\right) \\
& +a_{4} \sin \left(b_{4} t+c_{4}\right)+a_{5} \sin \left(b_{5} t+c_{5}\right)+a_{6} \sin \left(b_{6} t+c_{6}\right)
\end{aligned}
$$

In the model expression, $x=\left(a_{1}, b_{1}, c_{1}, a_{2}, b_{2}, c_{2}, a_{3}, b_{3}, c_{3}, a_{4}, b_{4}, c_{4}, a_{5}, b_{5}, c_{5}, a_{6}, b_{6}, c_{6}\right)$ is the model parameters to be solved, the number of model parameters $n=18, y$ is telemetry sampling data, $t$ is corresponding sampling time sequence.

3) Fourier series model 
The Fourier series model can fit well the data type of each periodic law, and is also a data model based on the harmonic frequency. Here is the model of the 4 order Fourier series, the model expression is as follows:

$$
\begin{aligned}
y=\Phi(x, t)= & a_{0}+a_{1} \cos (\omega t)+b_{1} \sin (\omega t)+a_{2} \cos (2 \omega t)+b_{2} \sin (2 \omega t)+ \\
& a_{3} \cos (3 \omega t)+b_{3} \sin (3 \omega t)+a_{4} \cos (4 \omega t)+b_{4} \sin (4 \omega t)
\end{aligned}
$$

In the model expression, $x=\left(a_{0}, a_{1}, b_{1}, a_{2}, b_{2}, a_{3}, b_{3}, a_{4}, b_{4}, \omega\right)$ is the model parameters to be solved, $\omega$ is Basic frequency, the number of model parameters $n=10, y$ is telemetry sampling data, $t$ is corresponding sampling time sequence.

4) exponential model

Exponential model can better fit with the trend of changes in the index data, the expression is more concise than multi order polynomial model, but considering the index rose rapidly, we only consider the $1 \sim 2$ order exponential model. Here is the model of the 2 order Exponent, the model expression is as follows:

$$
y=\Phi(x, t)=a_{1} e^{b_{1} t}+a_{2} e^{b_{2} t}
$$

In the model expression, $x=\left(a_{1}, b_{1}, a_{2}, b_{2}\right)$ is the model parameters to be solved, the number of model parameters $n=4, y$ is telemetry sampling data, ${ }^{t}$ is corresponding sampling time sequence.

5) Gauss model

The Gauss model fits well with the data of the arch trend. Here is given the 3 order Gauss model, the model expression is as follows:

$$
y=\Phi(x, t)=a_{1} \exp \left(-\left(\frac{t-b_{1}}{c_{1}}\right)^{2}\right)+a_{2} \exp \left(-\left(\frac{t-b_{2}}{c_{2}}\right)^{2}\right)+a_{3} \exp \left(-\left(\frac{t-b_{3}}{c_{3}}\right)^{2}\right)
$$

In the model expression, $x=\left(a_{1}, b_{1}, c_{1}, a_{2}, b_{2}, c_{2}, a_{3}, b_{3}, c_{3}\right)$ is the model parameters to be solved, the number of model parameters $n=9, y^{y}$ is telemetry sampling data, $t_{\text {is corresponding sampling time }}$ sequence.

6) BP Neural network model

BP Neural network model can better fit the data of non periodicity and square wave periodicity. It is known that there is a hidden layer of the three layer BP network, which can effectively approximate to any continuous function. The three layer network includes input layer, hidden layer and output layer. Considering the requirement of the network to predict the generalization performance of the network, Network design should adhere to the principle of reducing the complexity of the network as much as possible, using the three layer BP network model to predict the chaotic time series, The input layer node only transmits the input signal to the hidden layer, The hidden layer of the hyperbolic tangent function of S type of data processing to the output node, output nodes are simple linear functions.

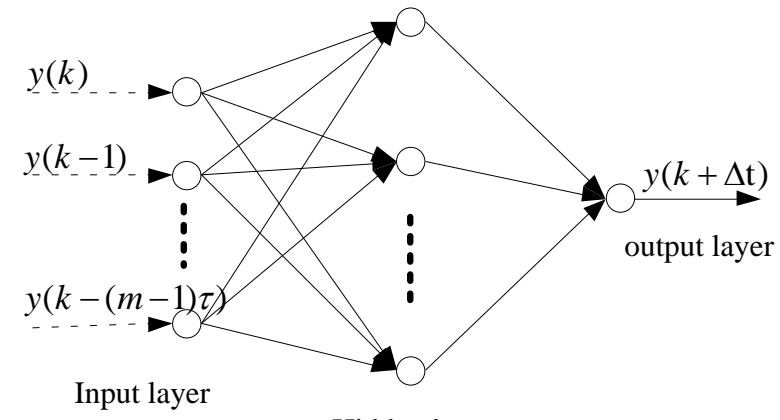

Hidden layer

Input data is: $Y(k)=[y(k), y(k-\tau), \cdots, y(k-(m-1) \tau)]$, output data is: $y(k+\Delta t)$, in the expression $k=1,2, \cdots, N, \mathrm{~m}$ is input dimension, $\tau$ is cross step.

In the application process, we obtain the original parameters of SumSin model and Fourier series model respectively based on FFT and QR decomposition; The parameters of the Exponent model and the of Gauss model are studied by the characteristics of the index data and the Gauss data. Using 
genetic algorithm and Levenberg-Marquardt algorithm, we can obtain accurate parameters of the SumSin model, Fourier series model, Exponent model, Gauss model and other models; Using BP algorithm to obtain the accurate parameters of neural network model.

\subsection{Forecast evaluation method}

Prediction and evaluation of the early warning system to assess the use of the implementation of the results of the assessment, making the results more accurate, as small as possible to deviate from the real situation. In the practice, we calculate the degree of implementation by the application of statistical principle.

1) Confidence statistical evaluation methods

First, we do data preprocess and analysis of the historical telemetry data (we set the history data set for the telemetry parameters is $\mathrm{H}=\{\mathrm{h} 1, \mathrm{~h} 2, \ldots \ldots \mathrm{hi}, \ldots . . \mathrm{hn}\}$ ), we get models and algorithms for data forecast. According to the model, the forecast value of the telemetry parameters is calculated (we set forecast data set for telemetry parameters is $\mathrm{P}=\{\mathrm{p} 1, \mathrm{p} 2, \ldots \ldots \mathrm{pi}, \ldots \ldots . \mathrm{pn}\})$, and compare it with the corresponding telemetry parameters $\mathrm{H}=\{\mathrm{h} 1, \mathrm{~h} 2, \ldots . . . \mathrm{hi}, \ldots . . . \mathrm{hn}\}$, and then work out the error set, $\mathrm{E}=\{\mathrm{e} 1, \mathrm{e} 2, \ldots . . . \mathrm{ei}, \ldots . . . \mathrm{en}\}$, according to the prior data analysis, a confidence degree is determined $\alpha \in(0,1]$. We define confidence interval value $\nabla$ as $100 * \alpha$ between $(\mathrm{H}-\nabla, \mathrm{H}+\nabla)$. The smaller the the $\alpha$ is, the value is closer to the truth. $\alpha=0$, means hi, the forecast value of telemetry data equals to pi, the corresponding forecast value, $\alpha=1$ means All values are credible. $(\mathrm{P}-\nabla, \mathrm{P}+\nabla)$ can be used as a confidence interval for the true value of the subsequent forecast value, the confidence interval is $\alpha$. By updating the historical telemetry parameter periodically, recalculating the predict value of the telemetry parameter, we can constant updating the confidence degree interval value $\nabla$, whose confidence degree is $\alpha$, so that we can be sure that the forecast value is within the latest and most accurate range.

2) Error statistics evaluation method

In the forecasting process, we can use the error statistical method in the statistical method to evaluate. We use the definite model and algorithm, according to historical data set of telemetry parameters (we define the historical data set of telemetry parameters as $\mathrm{H}=\{\mathrm{h} 1, \mathrm{~h} 2, \ldots . . \mathrm{hi}, \ldots . . . \mathrm{hn}\})$, we can get the forecast value of the required telemetry parameters(we define forecast value set of the telemetry parameters $\mathrm{P}=\{\mathrm{p} 1, \mathrm{p} 2, \ldots . . . \mathrm{pi}, \ldots . . . \mathrm{pn}\})$, we use the data of the set $H \& \mathrm{P}$, to calculate the data of the maximum absolute error, average error mean absolute error, we use the comparing benchmarks we set to analyze the mathematical statistics data, so we get the data to evaluate the system, which meet the user's request .

\subsection{Satellite borne equipment}

Through the summary and analysis of the computer on the application of the spacecraft in orbit, the software can be run in the following several environments:

1) Software environment: Operating system Vxworks, Satellite borne flash file system OFFS.

2) Hardware environment: Computers equipped with 3 different CUP, BM3803, PC8245, PC8548E.

Tab 2 list the CPU performance comparison among BM3803, PC8245, PC8548E.

Operating system Vxworks, satellite borne Flash file system OFFS, our institute has the research and development capabilities of the spacecraft related products, some related electronic products, developed on Vxworks, satellite borne Flash file system OFFS, have been tested on certain type of spacecraft, they work well and meet the design requirements.

BM3803 (domestic) is space level product, it has the index of anti radiation. PC8245 cannot provide space level product present, when it needs to be used in spacecraft, screening should be done. Related electronic products have been tested in orbit, the products work well and meet the design requirements; PC8548E cannot provide space level products present, when it needs to be used in spacecraft, screening should be done, and project demonstration is ongoing on certain spacecraft. 


\begin{tabular}{|c|c|c|c|}
\hline $\begin{array}{l}\text { Performance and } \\
\text { index }\end{array}$ & BM3803(domestic) & PC8245 & PC8548E \\
\hline Core & SPARC V8 & $\begin{array}{l}\text { G2(Derivative version } \\
\text { from PPC603e) }\end{array}$ & e500 \\
\hline Frequency & 100Mhz & 333Mhz & $1.5 \mathrm{Ghz}$ \\
\hline Performance & 86MIPS 23MFLOPS & 200 300MIPS & 3065MIPS@1333Mhz \\
\hline Cache & $\begin{array}{c}\text { Data Cache: } 16 \mathrm{~KB} \\
\text { Instruction Cache: } \\
16 \mathrm{~KB}\end{array}$ & $\begin{array}{l}\text { Instruction cache: } \\
16 \mathrm{~KB} \\
\text { Data cache: } 16 \mathrm{~KB}\end{array}$ & $\begin{array}{c}\text { 2level Cache: } \\
\text { L1: Instruction: } 32 \mathrm{~KB} \text {, } \\
\text { Data: } 32 \mathrm{~KB} \\
\text { L2: Instruction: } 512 \mathrm{~KB} \text {, } \\
\text { Data: } 512 \mathrm{~KB} \text {, }\end{array}$ \\
\hline $\begin{array}{l}\text { Error detection and } \\
\text { correction function }\end{array}$ & EDAC & $\mathrm{ECC}$ & ECC \\
\hline Memory interface & $\begin{array}{l}\text { PROM, FLASH, } \\
\text { SDRAM }\end{array}$ & $\begin{array}{l}\text { PROM, FLASH, } \\
\text { SDRAM }\end{array}$ & $\begin{array}{l}\text { DDR, DDR2, FLASH, } \\
\text { SDRAM }\end{array}$ \\
\hline $\begin{array}{l}\text { Peripheral interface } \\
\text { bus }\end{array}$ & PCI2.2 interface & PCI & $\begin{array}{c}2 \text { PCI or PCI-X }+1 \text { 个 } \\
\text { PCI-E }\end{array}$ \\
\hline Power & 1W@100MHz & 2.3W Typical & $\begin{array}{c}5 \mathrm{~W} @ 1000 \mathrm{MHz} \mathrm{Tj}=65^{\circ} \mathrm{C} \\
\text { Typical }\end{array}$ \\
\hline Device level & Space level & $\begin{array}{l}\text { Up screening(Atmel } \\
\text { orE2V) }\end{array}$ & $\mathrm{M}(\mathrm{E} 2 \mathrm{~V})$ \\
\hline Support Vxworks & yes & yes & yes \\
\hline
\end{tabular}

\subsection{Massive data storage research}

The basis of the early warning system is historical data, if we make a long time forecast, a large amount of historical data is needed as the system forecast input.

For a long period of time, a large amount of historical data is needed to forecast the input. Therefore massive data storage device is needed to store the historical telemetry data. We can obtain the required information from the large capacity memory in the process of running the system. In the early of the system design, data volume and data storage analysis should be done, so that we can select memory, and the access method for telemetry data.

1) Data quantity evaluation

At present, when the forecast evaluation algorithm of telemetry parameter early warning system, it is more close to the trend of telemetry parameters, it takes the historical telemetry data of 3 months at most, to forecast the future telemetry data for less than 1 month. According to the statistical data of XX-2 satellite, the quantity of all the 1632 telemetry data of 3mongth is about 55.1G, if we use the original code storage, the amount of data can be reduced to $1 / 8$ of the original. Comparing with the other data information, such as historical picture data and etc, telemetry information has the characteristic of low data transmitting rate, easy to be compressed and high compressed rate. So we can take compression algorithm in data storage to save storage space to increase the storage capacity for telemetry. We can use zlib algorithm, which has noting to do with the platform and easy to be transplanted, to store telemetry data. The algorithm uses the DEFLAE algorithm, LZ77 algorithm and Huffman encoding, it has mature technology, the lossless compression effect is good and the compression rate is at least 0.02 . However, when using the compression algorithm, it may affect the efficiency and speed of data retrieval, which has a certain influence on the performance and real-time performance of the system.

2) Memory evaluation

Fast recording technology of massive data has been applied in the domestic and abroad, our institute has put the massive data storage unit of $80 \mathrm{G}$ on XX-2. At present, pre research on fast data storage prototype has been carried out, and the prototype has been completed (fig 2). Prototype technology index has reached the speed of reading and storing at the rate of $5 \mathrm{Gbps}$, the Capacity is about $2.56 \mathrm{~Tb}$ 
and it can be extended; the prototype machine support documented data management, by establishing an index table for storing files so that, the machine can locate the storage data quickly, the responding time is within $0.2 \mathrm{~s}$, and it has function of file index, which greatly improve the performance of early warning system.
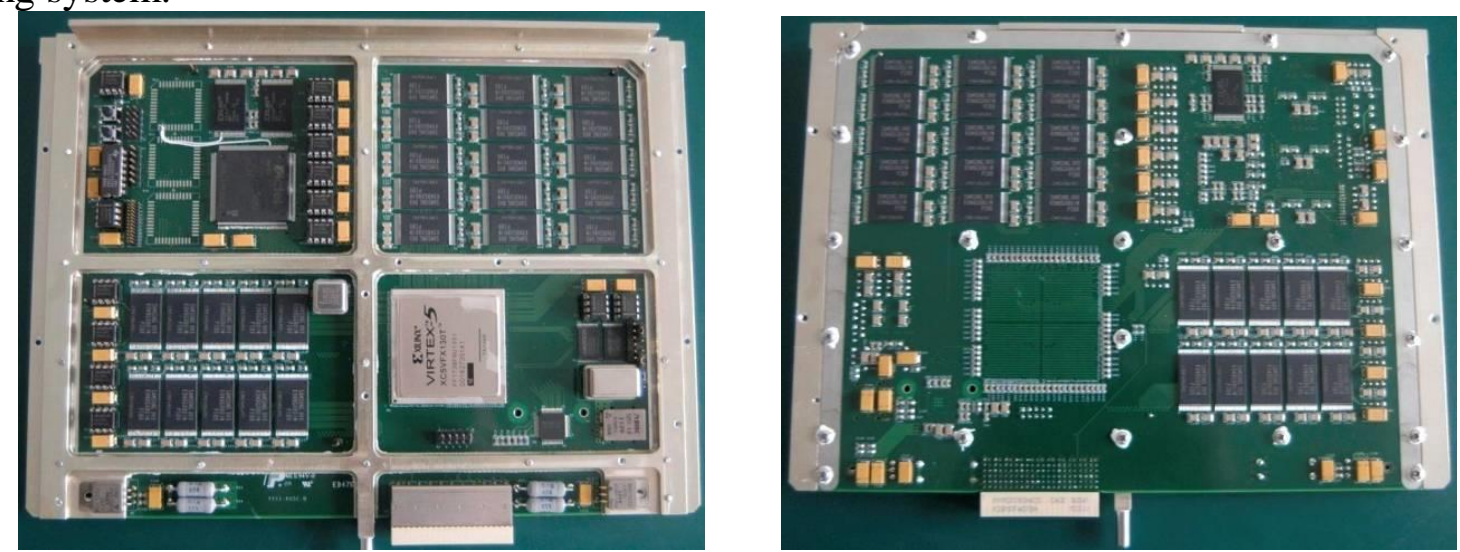

Fig 2 Principle prototype of high speed data memory

\section{Early warning system}

\subsection{General planning}

According to the requirement analysis, and basing on the technology and method of engineering practice, the early warning system can be divided into several requirement modules. They are configuration information management, telemetry parameter prediction, early warning, forecast task allocation, information display, external interface management and database interface. By main thread scheduling, they realize the user function of the forecast information management, telemetry parameters forecasting, early warning and interactive interface management. Structure diagram is as fig3 shows.

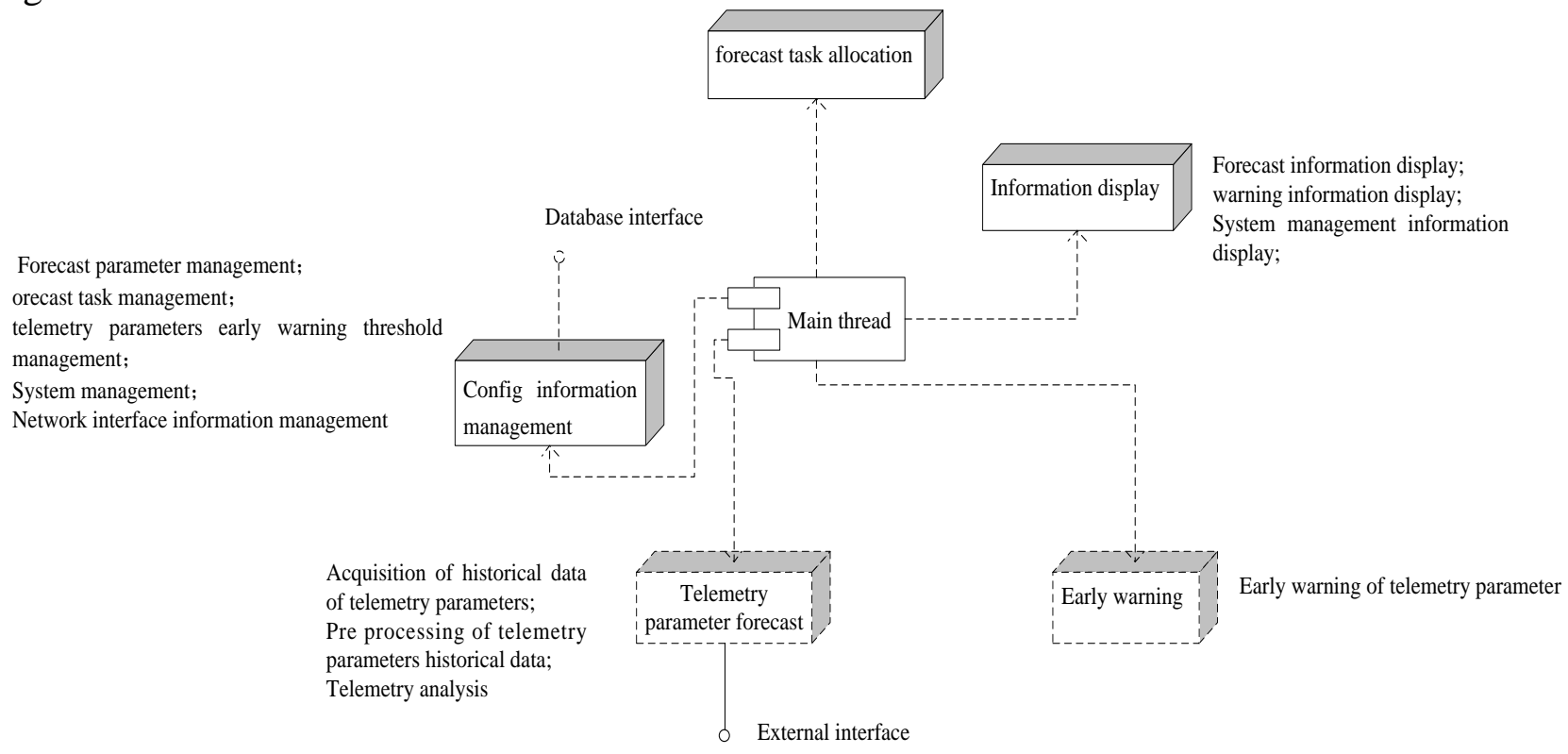

Fig 3 System structure diagram

Telemetry database manage system receives real time telemetry data information, and stores in database in chronological order, if the data to be stored is larger than the storage capacity assumed, the historical data that first stored should be erased. Forecasting of task allocation and center control subsystem launched an early warning tasks and the task information is sent to the data receiving subsystem. Data receiving and distributing subsystem according to the received early warning task and obtain the corresponding telemetry historical data information and distribute data information to the telemetry parameters prediction subsystem. The telemetry parameters forecast subsystem complete the 
information acquisition of historical telemetry data information, and then preprocess the historical telemetry data information, forecast the task allocation(input information of predictive algorithm model), process the historical telemetry data information, calculate the forecast telemetry data information, analyze the forecast telemetry data information, and then carry out the calculate the execution degree of the forecast information. The early warning management subsystem obtains the forecast data and the early warning threshold profile from the forecast task allocation and the central control subsystem, and completes comparing the theoretical threshold of the telemetry parameters, the confidence level and the user set up. When the forecast value deviates from the confidence level or beyond the theoretical threshold, the real-time warning information is given, and the corresponding information is transmitted to the forecasting task allocation and the central control subsystem. Forecast task allocation and central control subsystem transmit the predict results to the required control terminal through the network or bus, used in orbit management, fault diagnosis and other functions.

\subsection{Early warning system}

In early warning system, the least square polynomial method, LM+ Harmonic method, GA+Fourier method, BP neural network method and LM+ Index method are used. All the method can do forecast of telemetry data for spacecraft in orbit, we get 20000 data set. The manners and testing performance is as tab 3 shows. The time of getting data depends on the complex of storing the data, and the predict time depends on the complex of algorithm.

Table 3 statistics form

\begin{tabular}{cccc}
\hline Algorithm name & Applicable data type & $\begin{array}{c}\text { Data acquisition } \\
\text { time(s) }\end{array}$ & $\begin{array}{c}\text { Forecast } \\
\text { time(s) }\end{array}$ \\
\hline $\begin{array}{c}\text { Least squares } \\
\text { polynomial }\end{array}$ & $\begin{array}{c}\text { With the simple monotonic change law is } \\
\text { mainly applied to linear changes. }\end{array}$ & 3.2 & 1.1 \\
\hline SumSin model & Has many kinds of superimposed periodic \\
variation & 5.9 & 16.8 \\
\hline GA+Fourier & Simple periodic variation & 4.1 & 21.1 \\
\hline BPNeural network & With complex changes & 4.0 & 29.3 \\
\hline Index method & Long monotone nonlinear variation law & 8.0 & 7.9 \\
\hline
\end{tabular}

Take four kinds of typical forecast values for the results show, as is shown in figure 4, the red line represents the upper and lower threshold of the telemetry parameters, black line represents the value of historical telemetry value, purple line represents the forecast telemetry parameter, green point represents the real time value of telemetry parameter.

From figure 4, we can see that the trend is basically consistent with the actual data, and it can track parameter curve well.

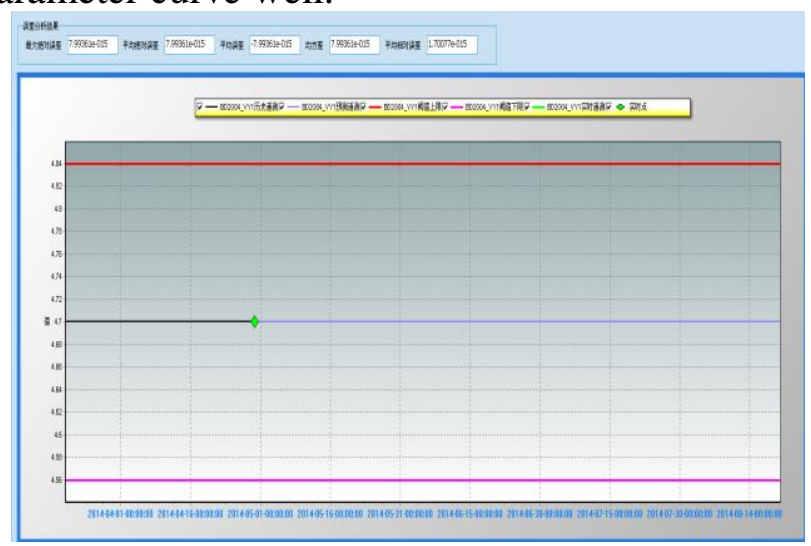

Fig.4-1 Least squares polynomial

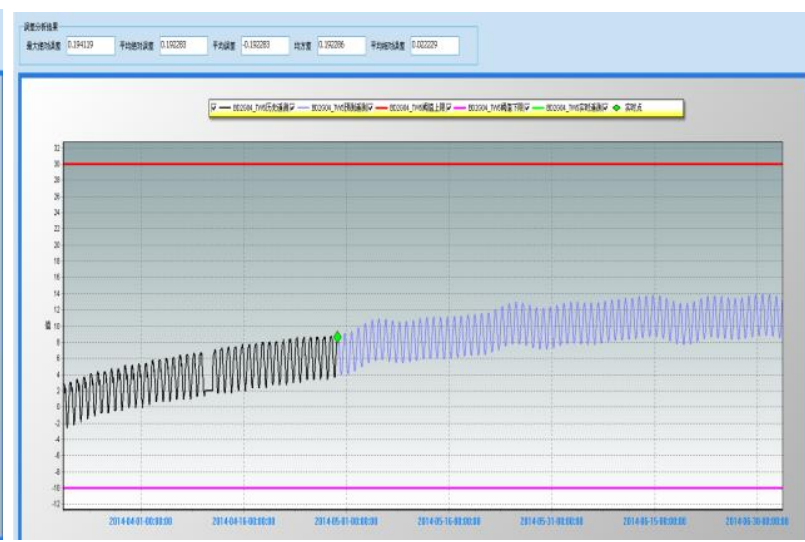

Fig.4-2 SumSin model 


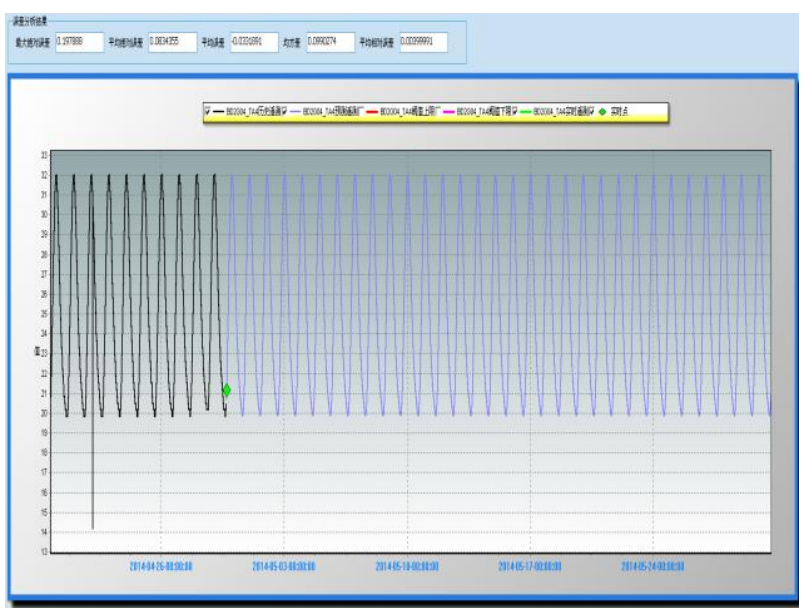

Fig.4-3 GA+Fourier

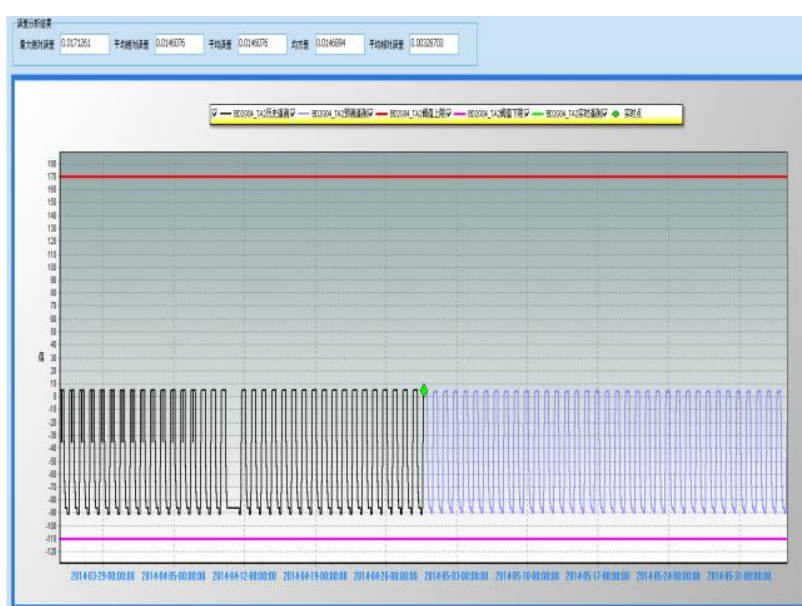

Fig.4-4 BP Neural network

Table 4 Forecast error analysis table

\begin{tabular}{cccccc}
\hline Algorithm name & $\begin{array}{c}\text { Maximum } \\
\text { absolute error }\end{array}$ & $\begin{array}{c}\text { Mean absolute } \\
\text { error }\end{array}$ & Mean error & $\begin{array}{c}\text { Mean square } \\
\text { deviation }\end{array}$ & $\begin{array}{c}\text { Mean relative } \\
\text { error }\end{array}$ \\
\hline $\begin{array}{c}\text { Least squares } \\
\text { polynomial }\end{array}$ & $7.9936 \mathrm{E}-15$ & $7.9936 \mathrm{E}-15$ & $-7.9936 \mathrm{E}-15$ & $7.9936 \mathrm{E}-15$ & $1.70077 \mathrm{E}-15$ \\
\hline SumSin model & 0.194119 & 0.192283 & 0.192283 & 0.192286 & 0.022222 \\
\hline GA+Fourier & 0.197888 & 0.083485 & 0.033199 & 0.099027 & 0.003999 \\
\hline $\begin{array}{c}\text { BP Neural } \\
\text { network }\end{array}$ & 0.017261 & 0.014507 & 0.014507 & 0.014689 & 0.003287 \\
\hline
\end{tabular}

Forecasting and monitoring of early warning system is shown in figure 5,the blue point and dash line represents the prefabricated the user sets, red line represents the confidence interval, purple line represents the actual value, green line represents the predict value. When the forecast value exceeds the preset range of the user sets, system alarms and outputs the corresponding information to other systems.

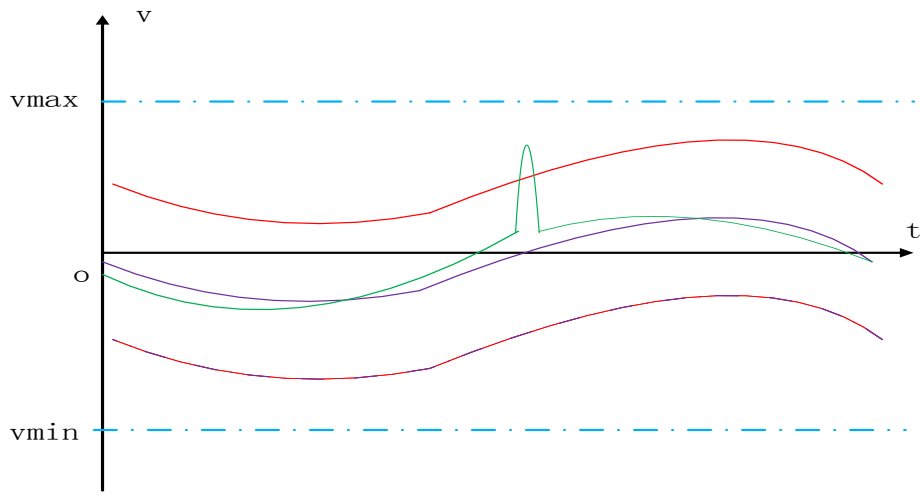

Fig. 5 monitor of early warning system

\section{Summary}

The early warning system of satellite telemetry data has been realized in the ground support system, and it can carry out the function of trend forecasting and early warning of telemetry data. The system has already accumulated amount of experience and method to process, forecast and make early warning. If the system will be equipped in spacecraft, we should verify the availability of the forecast algorithm and the early warning system of satellite telemetry data in spacecraft environment by a prototype, which is made by BM3803 (domestic), PC8245, PC8548E CPU and massive memory.

\section{References}

[1] Zhang Bangxin, Suo Xunhua. Technique for diagnosing satellite failure [C]// 2001 Academic 
Conference for Simulation Technique. Beijing: China Computer Users Association, 2001: 213-215 (in Chinese)

[2] Tan Weizhi, Hu Jingang. Spacecraft systems engineering [M]. 2nd Edition. Beijing: China Science and Technology Press, 2009 (in Chinese)

[3] Caceres M. Satellites and launches trend down [J]. Aerospace America, 2004, 42(1): 18-20

[4] Zhang Yanduo, Jiang Xinwei, Huang Wenhu. Design of satellite TM system for fault simulator and alarm [J]. Journal of Vibration, Measurement \& Diagnosis, 2002,22(3): 186-189 (in Chinese)

[5] Roemer M J, Byington C S, Kacprzynski G J, et. An overview of selected prognostic technologies with reference to an integrated PHM architecture [C]. Proceedings of IEEE Aerospace. 2005:1 15

[6] Al-Smadi, A. The estimation of the order of an ARMA process using third-order statistics. International Journal of Systems Science vol. 36(15):975 980, Dec. 2005

[7] Richard C, Millar. A Systems Engineering Approach to PHM for Military Aircraft Propulsion Systems [M]. NAVAIR Propulsion \& Power, 2007

[8] Rrook R S, David L A. A survey of serviceable spacecraft failures, AIAA-2001-4540 [R]. Washington: AIAA, 2001 